Ann. Génét. Sél. anim., I969, 1 (I), 49-65.

\title{
SÉLECTION DIFFÉRENTE DANS LES DEUX SEXES. DISCUSSION GÉNÉRALE DES POSSIBILITÉS D'ÉQUILIBRE POUR UN LOCUS AUTOSOMAL A DEUX ALLELLES
}

\author{
P. MÉRAT \\ Station centrale de Génétique animale, \\ Centre national de Recherches zootechniques, 78 -Jouy-en-Josas \\ Institut national de la Recherche agronomique
}

SOMMAIRE

Nous avons passé en revue, dans le cas général, les possibilités d'équilibre des fréquences géniques, pour un locus autosomal à deux allèles en panmixie, lorsque la sélection diffère dans les deux sexes. Ces possibilités sont les suivantes :

- Absence d'overdominance : aucun équilibre autre que la fixation de A ou de $a$ si le classement des génotypes est identique dans les deux sexes; 0,1 ou 2 équilibres si le classement des homozygotes s'inverse d'un sexe à l'autre. Lorsqu'il est unique, l'équilibre peut être stable ou instable suivant le cas, à en juger par la stabilité au voisinage de la fixation de $A$ ou de $a$.

- Overdominance positive dans un sexe, absente dans l'autre : 0 ou 1 équilibre.

- Overdominance positive dans les deux sexes : 1 équilibre (stable) à en·juger par l'instabilité au voisinage de la fixation de $\mathbf{A}$ ou de $a$.

- Overdominance positive dans un sexe, négative dans l'autre : $0,1,2$ ou 3 équilibres.

- Overdominance absente dans un sexe, négative dans l'autre : 0,1 ou 2 équilibres.

- Overdominance négative dans les deux sexes : 1 équilibre (instable) à en juger par la stabilité au voisinage de la fixation de $\mathrm{A}$ ou de $a$.

Ces résultats confirment donc en particulier la possibilité, dans certains cas, de deux équilibres, dont un pouvant être stable, et montrent que l'existence de trois équilibres, dont deux pouvant être stables, n'est possible que lorsque l'hétérozygote est favorisé dans un sexe et défavorisé dans l'autre par rapport aux deux homozygotes.

\section{INTRODUCTION}

Il n'est pas rare que la valeur sélective des divers génotypes à un locus, vis-àvis de la sélection naturelle ou artificielle, diffère suivant le sexe. Un cas particulier courant est celui des gènes à expression limitée à un sexe ou modifiée par le sexe, si cette expression englobe un ou plusieurs caractères soumis à une sélection $\left(^{1}\right)$.

De même, les coefficients de sélection sont différents chez les mâles et les femelles pour un gène responsable à la fois d'un effet maternel et d'un effet

(1) Dans le cas de l'intersexualité liée au gène " sans cornes " chez la chèvre, les différences des valeurs sélectives entre sexes sont extrêmes (I,AUVERGNE, à paraître). 
direct sur le phénotype de son possesseur (MÉRAT, I966), ou pour un gène influant sur la variance phénotypique, en sélection artificielle, du fait des différences d'intensité de sélection exercées dans les deux sexes (MÉRaT, r967).

Parmi les études théoriques sur la possibilité d'équilibre des fréquences géniques dans ce cas, les premières en date semblent celles de HALDANE (I924, 1926), qui passe en revue certains cas particuliers et donne une solution approximative lorsque les coefficients de sélection sont petits. OWEN (I953) amorce l'étude du cas général. Il conclut à la possibilité, parfois, de trois fréquences d'équilibre, dont deux correspondent à des équilibres stables. BODMER (I965) reprend cette étude et examine la situation spéciale où le rapport des différences de viabilité (homozygote-hétérozygote) est le même dans les deux sexes. D'autres modèles simplifiés ont été analysés par Parsons (I96r, gène de faible fréquence initiale), HALDANE (rg62), Li (r963, génotype homozygote létal dans les deux sexes).

Une discussion complète du cas général n'a pas été faite, à notre connaissance, sans doute à cause de la forme algébriquement peu simple des équations obtenues.

Notre propos est précisément d'examiner en détail, dans les différentes situations génétiques concevables, la possibilité et éventuellement le nombre d'équilibres des fréquences géniques que l'on peut obtenir, à partir de l'équation donnée par OWEN, cet auteur ayant essentiellement limité sa discussion à la possibilité de réalisation de trois équilibres.

Nous n'envisagerons pas le cas des gènes liés au sexe, déjà traité (HALDANE, I926; Sprotr, I957; BennetT, I958; Mander, I959; Halddane et Jayakar, I964).

\section{FORMULATION DU CAS GÉNÉRAL}

Nous rappellerons brièvement les résultats obtenus par OWEN (I953), en y ajoutant quelques précisions qui nous seront utiles pour notre discussion ultérieure.

Supposons une population d'effectif illimité ou très grand, en panmixie, avec les allèles $\mathrm{A}$ et $a$ au locus autosomal $\mathrm{A}$, et les fréquences de départ indiquées au tableau $I$, les générations étant discontinues, sans recouvrement. L'on peut considérer ces fréquences comme celles existant chez les reproducteurs des deux sexes qui contribuent effectivement à la génération suivante. Elles sont synonymes, dans ce modèle déterministe, des probabilités d'apparition des allèles.

TABLEAU I

Fréquences de départ des deux allèles

\begin{tabular}{|c|c|c|c|}
\hline Sexe & & ces & $\begin{array}{c}\text { Rapport } \\
\text { des fréquences }\end{array}$ \\
\hline †? & $p_{1}$ & $q_{1}$ & $u_{1}=\frac{p_{1}}{q_{1}}$ \\
\hline $0^{7} \sigma^{\circ}$ & $p_{2}$ & $q_{2}$ & $u_{2}=\frac{p_{2}}{q_{2}}$ \\
\hline
\end{tabular}


Le tableau 2 indique les " coefficients de sélection " s'appliquant aux différents génotypes dans les deux sexes. Ces coefficients sont liés à la probabilité, pour un génotype dans un sexe, de contribuer à la formation de la génération suivante.

\section{TABLEAU 2}

Coefficients de sélection des divers génotypes

\begin{tabular}{|c|c|c|c|}
\hline \multirow{2}{*}{ Sexe } & \multicolumn{3}{|c|}{ Génotypes } \\
\hline & AA & $\mathrm{A} a$ & $a a$ \\
\hline 우우 & $I-S_{1}$ & I & $\mathrm{I}-s_{1}$ \\
\hline$d \sigma^{*}$ & $I-S_{2}$ & I & $\mathrm{I}-s_{2}$ \\
\hline
\end{tabular}

Les paramètres tels que $S_{1}$ peuvent être positifs (ils sont alors inférieurs à $I$ ) ou négatifs.

Le tableau 3 donne les proportions des génotypes après opération de la sélection sur les zygotes issus de la génération de départ. Les fréquences ou probabilités sont égales aux facteurs figurant dans le tableau, divisés par la somme de ces facteurs pour chaque sexe.

\section{TABLEAU 3}

Proportion des génotypes, par sexe, après sélection

\begin{tabular}{|c|c|c|c|}
\hline & $\mathbf{A A}$ & $\mathbf{A} a$ & $a a$ \\
\hline $\begin{array}{l}\text { oqo } \\
\text { कृ }\end{array}$ & $\begin{array}{l}\left(\mathrm{I}-\mathrm{S}_{1}\right) p_{1} p_{2} \\
\left(\mathrm{I}-\mathrm{S}_{2}\right) p_{1} p_{2}\end{array}$ & $\begin{array}{l}p_{1} q_{2}+p_{2} q_{1} \\
p_{1} q_{2}+p_{2} q_{1}\end{array}$ & $\begin{array}{l}\left(\mathbf{I}-s_{1}\right) q_{1} q_{2} \\
\left(\mathbf{I}-s_{2}\right) q_{1} q_{2}\end{array}$ \\
\hline
\end{tabular}

Si $p_{1}^{\prime}, q_{1}^{\prime}, p_{2}^{\prime}, q_{2}^{\prime}$ sont respectivement les fréquences des allèles $\mathrm{A}$ et $a$ dans les deux sexes parmi les zygotes du tableau 3 , en posant $u_{1}^{\prime}=\frac{p_{1}^{\prime}}{q_{1}^{\prime}}, u_{2}^{\prime}=\frac{p_{2}^{\prime}}{q_{2}^{\prime}}$, on a :

$$
\begin{gathered}
u_{1}^{\prime}=\frac{\left(\mathrm{I}-\mathrm{S}_{1}\right) u_{1} u_{2}+\frac{\mathrm{I}}{2}\left(u_{1}+u_{2}\right)}{\left(\mathrm{I}-s_{1}\right)+\frac{\mathrm{I}}{2}\left(u_{1}+u_{2}\right)} \\
u_{2}^{\prime}=\frac{\left(\mathrm{I}-\mathrm{S}_{2}\right) u_{1} u_{2}+\frac{\mathrm{I}}{2}\left(u_{1}+u_{2}\right)}{\left(\mathrm{I}-s_{2}\right)+\frac{\mathrm{I}}{2}\left(u_{1}+u_{2}\right)}
\end{gathered}
$$


Un état d'équilibre suppose que $u_{1}^{\prime}=u_{1}$ et $u_{2}^{\prime}=u_{2}$. En posant $t=\frac{u_{2}}{u_{1}}$, on trouve que les conditions d'équilibre correspondent à :

$$
u_{1}=\frac{t-c_{1}}{\mathrm{I}-\mathrm{C}_{1} t} \quad u_{2}=\frac{c_{2} t-\mathrm{I}}{\mathrm{C}_{2}-t}\left(^{*}\right)
$$

avec

$$
\begin{array}{ll}
c_{1}=\mathrm{I}-2 \mathrm{~s}_{1} & c_{2}=\mathrm{I}-2 \mathrm{~s}_{2} \\
\mathrm{C}_{1}=\mathrm{I}-2 \mathrm{~S}_{1} & \mathrm{C}_{2}=\mathrm{I}-2 \mathrm{~S}_{2}
\end{array}
$$

En portant les expressions trouvées pour $u_{1}$ et $u_{2}$, par exemple, dans $t=\frac{u_{2}}{u_{1}}$, on voit que $t$ satisfait à l'équation du troisième degré :

$$
f(t)=t^{3}-3 \alpha t^{2}+3 \beta t-\mathrm{I}=0
$$

avec $\quad \alpha=\frac{\mathrm{I}}{3}\left(\mathrm{C}_{1} c_{2}+c_{1}+\mathrm{C}_{2}\right) \quad \beta=\frac{\mathrm{I}}{3}\left(\mathrm{C}_{2} c_{1}+c_{2}+\mathrm{C}_{1}\right)$.

La discussion de cette équation doit tenir compte du fait que $f(0)=-\mathbf{I}$, et que $f(t)$ est équivalent à $t^{3}$ quand $t \rightarrow \pm \infty$. Il y a au moins une racine. Il n'y en a qu'une, et elle est positive, en l'absence d'un maximum et d'un minimum pour $f(t)$.

L'existence de trois racines positives suppose la réalité de ce maximum et de ce minimum, c'est-à-dire que $f^{\prime}(t)$ puisse s'annuler, donc que $\alpha^{2}-\beta \geqslant 0$. Il faut aussi $\alpha>0$ et $\beta>0$ pour que le maximum de gauche corresponde à $t>0$.

D'autre part, il est nécessaire que les valeurs de $f(t)$ correspondant au maximum et au minimum soient de signes contraires, condition exprimée par OWEN de la façon suivante :

$$
4 \alpha^{3}+4 \beta^{3}-3 \alpha^{2} \beta^{2}-6 \alpha \beta+\mathrm{I}<0
$$

On peut ajouter que ceci n'est réalisable que pour $\alpha$ et $\beta$ tous deux supérieurs à $+\mathbf{I}$. La condition (2) est donc en fin de compte la seule à prendre en considération, puisqu'elle englobe les précédentes.

Dans le plan de coordonnées $\alpha$ et $\beta$, la figure I schématise la zone unique dans laquelle trois racines positives existent. On vérifie que cette zone englobe la droite $\alpha=\beta$ (pour $\alpha$ et $\beta$ supérieurs à $+\mathrm{r}$ ) et présente une symétrie par rapport à cette droite (du fait que la condition (2) est symétrique par rapport à $\alpha$ et $\beta$ ).

Mais cette condition ne suffit pas pour qu'il existe trois équilibres géniques. Il faut, en outre, que les rapports $u_{1}$ et $u_{2}$ correspondant à un état d'équilibre soient positifs :

$$
\frac{t-c_{1}}{\mathrm{I}-\mathrm{C}_{1} t}>0 \quad \text { (3) } \quad \text { et } \quad \frac{c_{2} t-\mathrm{I}}{\mathrm{C}_{2}-t}>0
$$

(*) Et non $u_{2}=\frac{c_{2} t-1}{C_{9} t-t^{2}}$ comme indiqué par erreur dans l'article d'OwEN. 
$\mathrm{Si} \mathrm{C}_{1}>0$, la condition (3) implique que $t$ soit compris dans l'intervalle $\left(c_{1}, \frac{\mathrm{I}}{\mathrm{C}_{1}}\right)^{(*)}$; si $\mathrm{C}_{1}<0, t$ doit être supérieur à la fois à $\frac{\mathrm{I}}{\mathrm{C}_{1}}$ (déjà contenu par ailleurs darıs la condition $t>0$ ) et à $c_{1}(* *)$.

La condition (4) est nécessairement réalisée si (2) et (3) le sont, mais nous l'utiliserons dans la discussion qui suit. Si $c_{2}>0$, elle implique que $t$ soit inclus dans l'intervalle $\left(\mathrm{C}_{2}, \frac{\mathrm{I}}{c_{2}}\right)$; si $c_{2}<0$, elle revient à $t$ supérieur à $\frac{\mathrm{I}}{c_{2}}$ (contenu dans la condition $t>0$ ) et à $\mathrm{C}_{2}$.

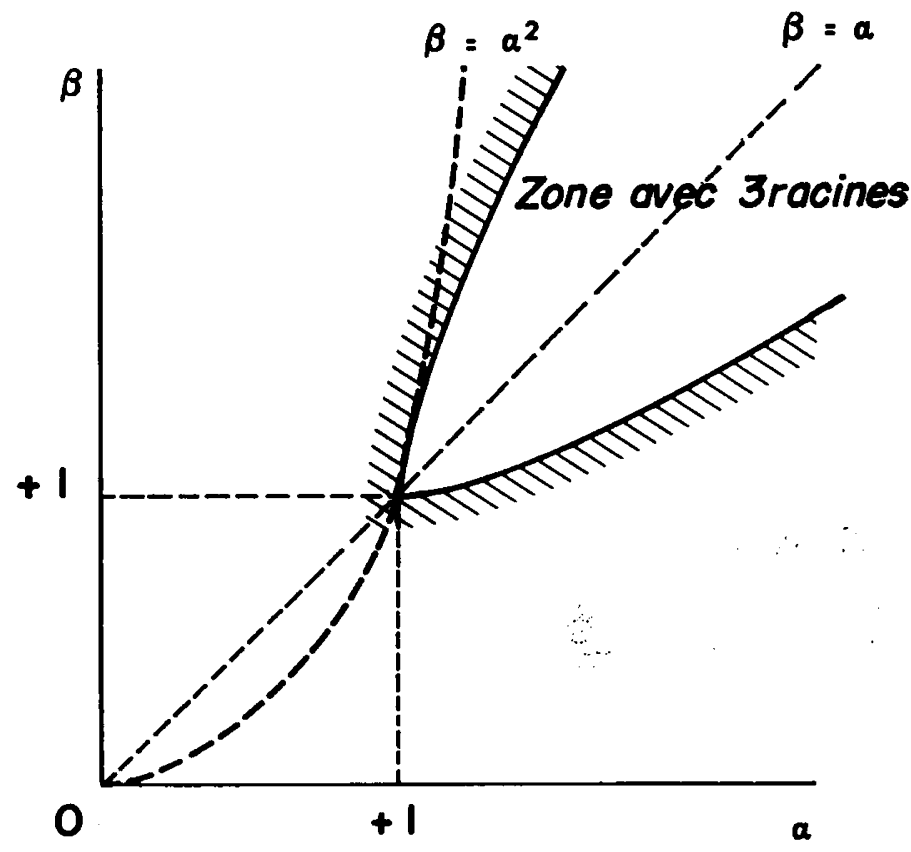

Fic. I. - Existence d'équilibres suivant les paramètres $\alpha$ et $\beta$

\section{LES POSSIBILITÉS D'ÉQUILIBRE SUIVANT LA SITUATION GÉNÉTIQUE}

En partant, comme l'ont fait OWEN (1953) et BoDmer (1965), de la discussion directe de l'équation du $3^{\mathrm{e}}$ degré en $t$, les conditions pour obtenir $0, \mathrm{I}, 2$ ou 3 équilibres sont difficiles à interpréter dans le cas général.

Par contre, nous allons voir qu'en classant les valeurs des paramètres $S_{1}, S_{2}$, $s_{1}$ et $s_{2}$ suivant la situation génétique dans chaque sexe, la discussion conduit à une interprétation plus claire.

(*) $c_{1}$ pouvant être supérieur ou inférieur à $\frac{I}{C_{1}}$.

$\left(^{* *}\right)$ Cette seconde possibilité a été omise par Owen. 


\section{I. - Classification des situations du point de VUE de la sélection}

Nous distinguerons, dans chaque sexe, trois possibilités suivant le classement respectif des trois génotypes au locus A quant à leur " coefficient de sélection " défini au tableau 2 :

- A $a$ intermédiaire entre AA et aa (pas d'overdominance);

- A $a$ supérieur aux deux homozygotes (overdominance positive);

- Aa inférieur aux deux homozygotes (overdominance négative).

Ceci conduit aux situations distinctes suivantes :

a) Absence d'overdominance, avec classement des génotypes identique dans les deux sexes.

b) Absence d'overdominance avec classement différent dans les deux sexes :

$\mathrm{AA}>\mathrm{A} a>a a$ dans un sexe,

$a a>\mathrm{A} a>\mathrm{AA}$ dans l'autre.

c) Overdominance positive dans un sexe, pas d'overdominance dans l'autre :
$(\mathrm{A} a>\mathrm{AA}>a a$
$(\mathrm{AA}>\mathrm{A} a>a a$
ou
$(\mathrm{A} a>\mathrm{AA}>a a$,
$(a a>\mathrm{A} a>\mathrm{AA}$.

d) Overdominance positive dans les deux sexes.

e) Overdominance positive dans un sexe, négative dans l'autre :
$(\mathrm{A} a>\mathrm{AA}>a a$
$(\mathrm{AA}>a a>\mathrm{A} a$
ou
$(\mathrm{A} a>\mathrm{AA}>a a$,
$(a a>\mathrm{AA}>\mathrm{A} a$.

f) Pas d'overdominance dans un sexe, overdominance négative dans l'autre :
$(\mathrm{AA}>\mathrm{A} a>a a$
$(\mathrm{AA}>a a>\mathrm{A} a$
ou
$\mathrm{AA}>\mathrm{A} a>a a$,
$a a>\mathrm{AA}>\mathrm{A} a$.

g) Overdominance négative dans les deux sexes.

\section{2. - PRINCIPES DE LA DISCUSSION POUR CHAQUE CAS PARTICULIER}

Les trois possibilités envisagées (pas d'overdominance, overdominance positive ou négative) se traduisent par des limites imposées aux paramètres " $S$ ", donc aussi aux paramètres " $\mathrm{C}$ ".

Ainsi, l'absence d'overdominance chez les femelles, correspondant au classement $\mathrm{AA}>\mathrm{A} a>a a$, suppose $\mathrm{S}_{1}<\mathrm{o}$ et $\mathrm{s}_{1}>0$, d'où $\mathrm{C}_{1}>+\mathrm{I}$ et $c_{1}<+\mathrm{I}$.

La connaissance du classement, les unes par rapport aux autres, des valeurs $c_{1}$, $\frac{\mathrm{I}}{\mathrm{C}_{1}}, \mathrm{C}_{2}, \frac{\mathrm{I}}{\mathrm{C}_{2}}$, permettra d'abord de déterminer s'il peut exister un équilibre, ou, plus précisément, si les conditions (3) et (4) peuvent être satisfaites simultanément, ce qui requiert :

- si $\mathrm{C}_{1}$ et $c_{2}$ sont tous deux positifs, qu'il existe une partie commune aux deux intervalles $\left(c_{1}, \frac{I}{C_{1}}\right)$ et $\left(C_{2}, \frac{I}{c_{2}}\right)$; 
- si $C_{1}>0$ et $c_{2}<0$, qu'une partie au moins de l'intervalle $\left(c_{1}, \frac{I}{C_{1}}\right)$ soit supérieure à la plus grande des deux valeurs $\left(C_{2}, \frac{I}{c_{2}}\right)$. $c_{2}<$ o.

Le raisonnement sera analogue pour les cas $\mathrm{C}_{1}<0, c_{2}>0$ et $\mathrm{C}_{1}<0$

En outre, dans tous les cas les $C$ sont situés sans ambiguité par rapport à la valeur $+\mathrm{r}$. Ceci permet de déterminer parfois le signe de $u_{1}$ ou $u_{2}$ suivant que $t$ est supérieur ou inférieur à $\mathrm{I}$, et par ailleurs, de connaître sans ambiguité dans une grande partie des cas, le signe de $f(t)$ pour $t=c_{1}, \frac{I}{C_{1}}, C_{2}$ et $\frac{I}{c_{2}}$. En effet, l'on peut vérifier que $f(t)$ s'écrit :

$$
\begin{aligned}
& \text { - pour } t=c_{1}:\left(c_{1} c_{2}-\mathrm{I}\right)\left(\mathrm{I}-\mathrm{C}_{1} c_{1}\right), \\
& - \text { pour } t=\frac{\mathrm{I}}{\mathrm{C}_{1}}: \frac{\left(\mathrm{I}-\mathrm{C}_{1} c_{1}\right)\left(\mathrm{I}-\mathrm{C}_{1} \mathrm{C}_{2}\right)}{\mathrm{C}_{1}^{3}}, \\
& \text { - pour } t=\mathrm{C}_{2}:\left(\mathrm{C}_{1} \mathrm{C}_{2}-\mathrm{I}\right)\left(\mathrm{I}-c_{2} \mathrm{C}_{2}\right), \\
& - \text { pour } t=\frac{\mathrm{I}}{\mathrm{C}_{2}}: \frac{\left(\mathrm{I}-c_{1} c_{2}\right)\left(\mathrm{I}-\mathrm{C}_{2} c_{2}\right)}{\mathrm{C}_{2}^{3}}
\end{aligned}
$$

L'on pourra souvent aussi connaître le signe de $f(t)$ pour $t=+\mathrm{I}$, car, pour cette valeur, on a :

$$
f(t)=3(\beta-\alpha)=\left(I-c_{2}\right)\left(C_{1}-I\right)+\left(c_{1}-I\right)\left(C_{2}-I\right)
$$

De la sorte, on vérifiera la position de certaines racines de $f(t)$ par rapport aux valeurs $c_{1}, \frac{I}{C_{1}}, C_{2}, \frac{I}{c_{2}}$. Cette discussion très simple permet de délimiter les possibilités d'équilibre de façon complète dans beaucoup de cas, et au moins partielle dans les autres. Nous n'aurons à la compléter par des considérations plus élaborées que dans quelques situations.

Quant à la stabilité des équilibres, on peut, à partir des valeurs $\bar{u}_{1}$ et $\bar{u}_{2}$ caractérisant un équilibre, considérer de petites variations $x_{1}$ et $x_{2}$ telles que $u_{1}=\bar{u}_{1}+x_{1}$ et $u_{2}=\bar{u}_{2}+x_{2}$ et voir si les valeurs propres de la matrice faisant passer de $\left(x_{1}, x_{2}\right)$ à $\left(x_{1}^{\prime}, x_{2}^{\prime}\right)$ correspondant à la génération suivante sont inférieures ou non à l'unité (BODMER, I965). Dans le cas général, les conditions obtenues ne sont pas algébriquement simples et leur interprétation ne paraît pas aisée.

On peut obtenir une indication partielle en étudiant la stabilité des équilibres correspondant à la fixation de $\mathrm{A}$ ou de $a$. Considérons, au voisinage de ces équilibres, la fréquence de $p$ de $\mathrm{A}$ sur l'ensemble des deux sexes parmi les reproducteurs, ou parmi les zygotes issus de ces reproducteurs, à la conception :

$$
p=\frac{p_{1}+p_{2}}{2}
$$


Les fréquences des trois génotypes $\mathrm{AA}, \mathrm{A} a$ et $a a$ parmi les zygotes à la conception sont $p_{1} p_{2}, p_{3} q_{2}+p_{2} q_{1}, q_{1} q_{2}$. On peut montrer que ces fréquences sont celles qui correspondraient à la panmixie avec une fréquence $p$ parmi les reproducteurs des deux sexes, diminuées de $\frac{d^{2}}{2}=\frac{\left(p_{1}-p_{2}\right)^{2}}{4}$ pour chacun des génotypes homozygotes, et augmentées de $d^{2}$ pour l'hétérozygote.

Pour $p$ très voisin de o ou de $I, p_{1}$ et $p_{2}$ seront également très petits ou très proches de $\mathrm{I}$, et la variation $\Delta p$ de $p$ d'une génération à la suivante ne dépendra en première approximation que de $p$. Ainsi, au voisinage de $p=0$, en négligeant les infiniment petits du second degré, on a :

$$
p_{1} p_{2} \simeq 0, p_{1} q_{2}+p_{2} q_{1} \simeq 2 p, q_{1} q_{2} \simeq \mathrm{r}-2 p
$$

Il en serait de même, par symétrie, pour $p$ voisin de $\mathrm{I}$. Autrement dit, on a alors un $\Delta p$ unique, indépendant de $p_{1}-p_{2}$ en première approximation, et il est facile de déterminer la stabilité de l'équilibre correspondant à partir de ce $\Delta p$ unique. Si ce ou ces équilibres sont instables, cela montre la possibilité de non-fixation des allèles en cause, et suggère la stabilité d'au moins un équilibre intermédiaire.

\section{3. - Discussion de chaque situation}

a) Absence d'overdominance dans les deux sexes avec même classement des génotypes.

Supposons, par exemple, $S_{1}$ et $S_{2}$ positifs, $s_{1}$ et $s_{2}$ négatifs, c'est-à-dire :

$$
\begin{aligned}
& -\mathrm{I}<\mathrm{C}_{1}<\mathrm{I} \quad c_{1}>\mathrm{I}, \\
& -\mathrm{I}<\mathrm{C}_{2}<\mathrm{I} \quad c_{2}>\mathrm{I} \text {. }
\end{aligned}
$$

On voit alors que, pour $t<\mathrm{I}, u_{1}<0$, et que, pour $t>\mathrm{I}, u_{2}<0$. Aucun équilibre n'est possible pour une fréquence de $\mathrm{A}$ différente de o ou de $\mathrm{I}$.

b) Absence d'overdominance dans les deux sexes avec inversion du classement des homozygotes d'un sexe à l'autre.

On aura, par exemple :

$$
\mathrm{S}_{1}<0 \quad s_{1}>0 \quad s_{9}<0 \quad S_{2}>0
$$

d'où $\quad \mathrm{C}_{1}>\mathrm{I} \quad c_{1}<\mathrm{I} \quad c_{2}>\mathrm{I} \quad \mathrm{C}_{2}<\mathrm{I}$

On peut supposer $\frac{I}{c_{2}}<\frac{I}{C_{1}}$ saus perte de généralité ( $\left.{ }^{1}\right)$.

(1) Car dans le cas contraire, si l'on intervertit à la fois 1 et 2 et les grandes et petites lettres, on se retrouve dans la situation postulée ici. 
Pour qu'une racine de $f(t)=0$ soit comprise dans la partie commune aux intervalles $\left(\frac{I}{C_{1}}, c_{1}\right)$ et $\left(C_{2}, \frac{I}{c_{2}}\right)$, il ne faut pas que $\frac{I}{c_{2}}$ et $C_{2}$ soient tous deux inférieurs à $\frac{\mathrm{I}}{\mathrm{C}_{1}}$ et $c_{1}$, cas où cette partie commune t'existe pas et où aucun équilibre n'est, par suite, possible (cf exemples A et C de Bodmfr, 1965).

Cette condition peut s'interpréter de la façon suivante : les coefficients $S_{2}$ et $s_{2}$ de sélection ne doivent pas être, en valeur absolue, tous deux supérieurs ou tous deux inférieurs à $S_{1}$ et $s_{1}$, c'est-à-dire que la sélection dans un sexe ne doit pas être plus intense, sur les deux homozygotes à la fois, que dans l'autre sexe.

Ceci étant supposé réalisé, pour qu'une racine corresponde à un équilibre génique, il faut qu'elle soit comprise entre les deux valeurs intermédiaires du groupe des quatre valeurs $\left(c_{1}, \frac{\mathrm{I}}{\mathrm{C}_{1}}, \mathrm{C}_{2}, \frac{\mathrm{I}}{c_{2}}\right)$.

Le tableau 4 indique les différents ordres possibles de classement de ces quatre valeurs (uniquement dans le cas où les intervalles $c_{1}-\frac{\mathrm{I}}{\mathrm{C}_{1}}$ et $\mathrm{C}_{2}-\frac{\mathrm{I}}{c_{2}}$ ont une partie commune) et le signe correspondant de $f(t)$ pour chacune. On en déduit immédiatement les possibilités d'équilibre.

En fin de compte, on aura donc généralement o ou $x$ équilibre (un exemple de BODMER, I965, tabl. 4, p. 4I9, illustre notre cas $h$ ) et, dans une situation, jusqu'à deux équilibres. On peut vérifier qu'il en est ainsi pour les valeurs suivantes dess paramètres $\mathrm{C}: c_{1}=0, \mathrm{C}_{1}=2, c_{2}=3, \mathrm{C}_{2}=\mathrm{I}$.

De ces deux équilibres, BODMER a montré dans un cas particulier qu'un seul était stable, l'autre étant instable.

c) Overdominance positive dans un sexe; pas d'overdominance dans l'autre.

Le cas typique correspondra aux inégalités suivantes, respectivement, pour les $\mathrm{S}$ et pour les $\mathrm{C}$ :

$$
\begin{array}{llll}
\mathrm{S}_{1}<0 & s_{1}>0 & \mathrm{~S}_{2}>0 & s_{2}>0 \\
\mathrm{C}_{1}>\mathrm{I} & c_{1}<\mathrm{I} & \mathrm{C}_{2}<\mathrm{I} & c_{2}<\mathrm{I}
\end{array}
$$

Comme $\mathrm{C}_{1}>0$, une racine de $f(t)$, pour correspondre à un équilibre, doit être comprise dans l'intervalle $\left(c_{1}, \frac{I}{C_{1}}\right)$ qui est tout entier inférieur à $I$.

Il ne peut donc pas y avoir trois racines conduisant à un équilibre, puisqu'elles seraient toutes trois inférieures à $\mathbf{I}$ et que leur produit doit être égal à $I$.

De plus, on vérifie que, dans tous les cas, $f(+\mathrm{r})=\beta-\alpha$ est positif. On ne peut donc avoir non plus deux racines répondant à la question, ce qui nécessiterait $f(+\mathrm{I})<0$. Il y a donc, soit zéro, soit un équilibre des fréquences géniques.

Le tableau 5 indique laquelle de ces deux éventualités est réalisée, suivant la disposition relative des paramètres $\left(c_{1}, \frac{\mathrm{I}}{\mathrm{C}_{1}^{-}}, \mathrm{C}_{2}, \frac{\mathrm{I}}{c_{2}}\right)$. 
TABLEAU 4

Possibilités d'équilibre: cas oì il n'y a overdominance dans aucun sexe

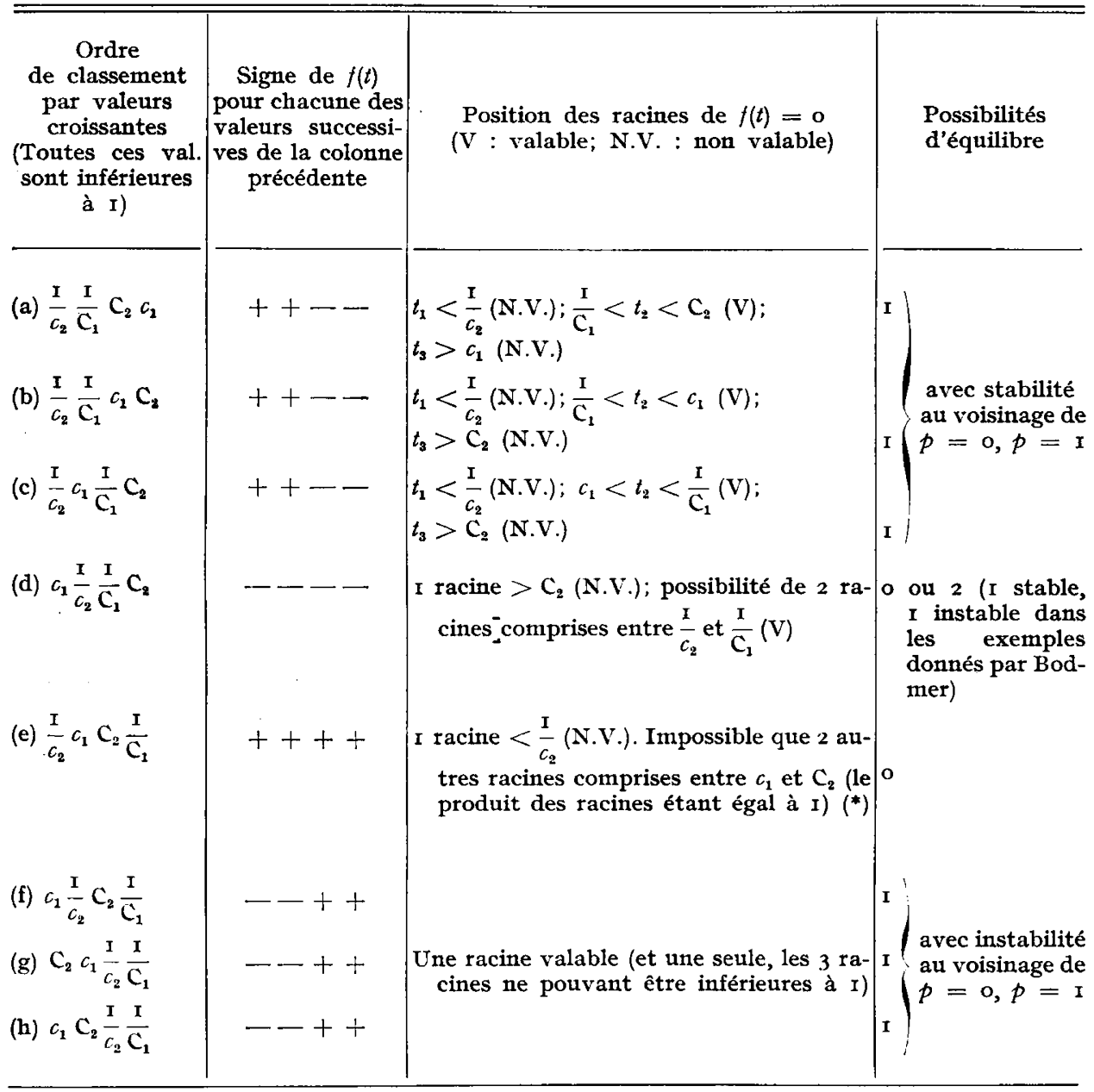

$\left(^{*}\right) f(t)$ pouvant s'écrire sous la forme $\left(t-t_{1}\right)\left(t-t_{2}\right)\left(t-t_{3}\right)$.

d) Overdominance positive dans les deux sexes.

Tous les paramètres $\mathrm{C}$ sont alors inférieurs à $\mathrm{I}$.

- Dans le cas où ils sont tous négatifs, il y a nécessairement au moins un équilibre, car alors, la racine positive $t$ qui existe toujours ne pourra pas être inférieure au plus grand des deux nombres $\left(c_{1}, \frac{\mathrm{I}}{\mathrm{C}_{1}}\right)$ et $\left(\mathrm{C}_{2}, \frac{\mathrm{I}}{c_{2}}\right)$. Cette situation correspond au choix, dans les deux sexes, de plus de $50 \mathrm{p}$. roo des reproducteurs parmi les hétérozygotes $\mathrm{A} a$. 
TABLEAU 5

Possibilités d'équilibre: cas oil il y a overdominance positive dans un sexe, pas d'overdominance dans l'autre

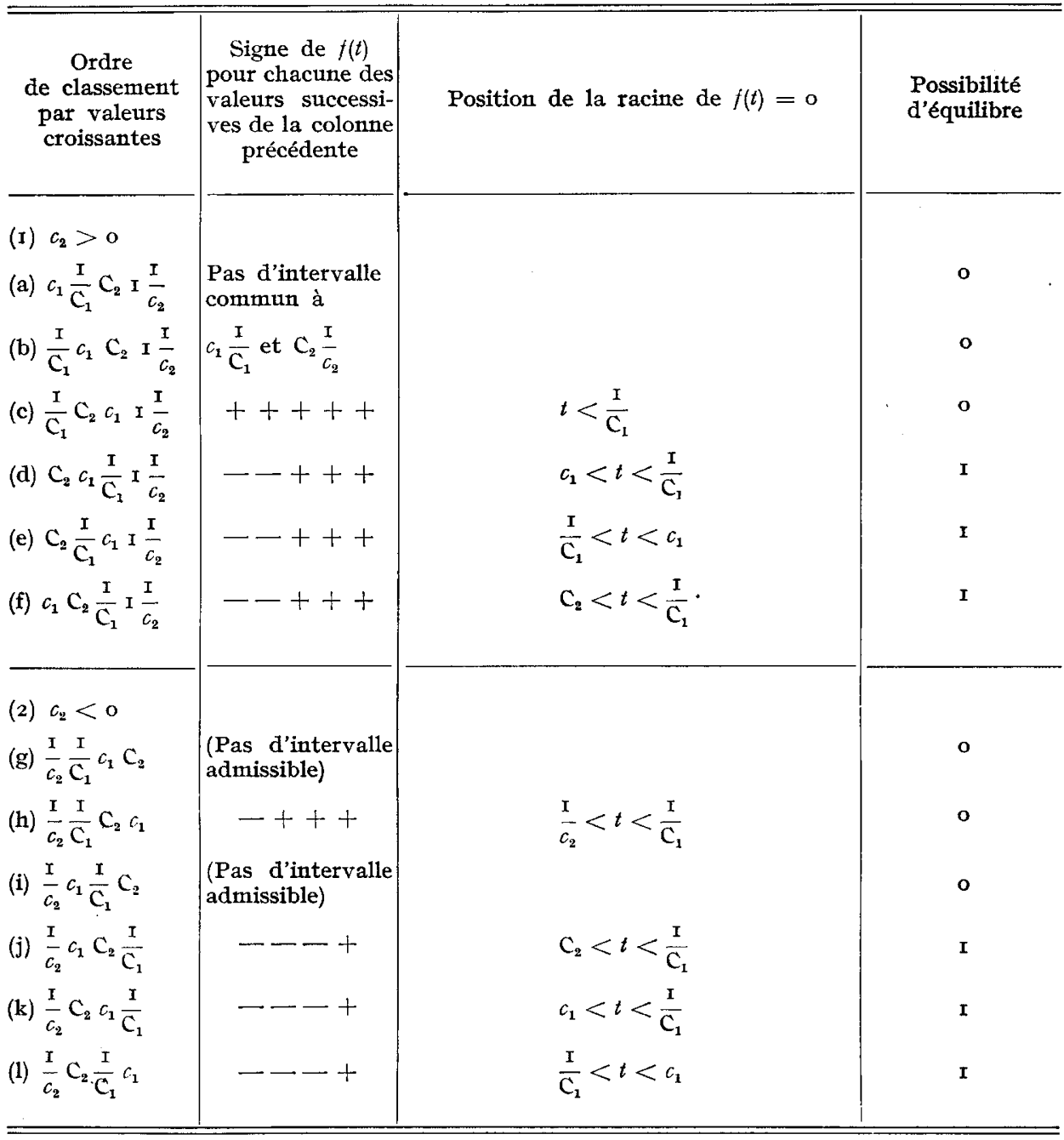

- Si tous les $\mathrm{C}$ sont positifs, $t$ doit se trouver à la fois dans les deux intervalles $\left(c_{1} \frac{I}{C_{1}}\right)$ et $\left(C_{2}-\frac{I}{c_{2}}\right)$. Ces deux intervalles ont obligatoirement une partie commune, car ils comprennent tous deux la valeur $+\mathbf{r}$.

On vérifie que $f(t)$ est négatif pour $t=c_{1}$ et $t=\mathrm{C}_{2}$, et positif pour $t=\frac{\mathrm{I}}{\mathrm{C}_{1}}$ et $t=\frac{\mathrm{I}}{c_{2}}$. Il y a donc au moins une racine de $f(t)=0$ comprise entre la plus grande des deux valeurs $\left(c_{1}, C_{2}\right)$ et le plus petit des deux nombres $\left(\frac{I}{C_{1}}, \frac{I}{c_{2}}\right)$. 
- Si certains des paramètres $\mathrm{C}$ sont négatifs, on vérifie de façon analogue que la racine positive existante correspond à un équilibre réel, car, dans tous les cas, $f(t)<0$ pour $t=c_{1}$ ou $\mathrm{C}_{2}$ et $f(t)$ est du signe, respectivement, de $\mathrm{C}_{1}$ ou de $c_{2}$ pour $t=\frac{\mathrm{I}}{\mathrm{C}_{1}}$ et $t=\frac{\mathrm{I}}{c_{2}}$, de sorte que la racine en question se trouve supérieure à $\mathrm{C}_{1}$ et $\frac{\mathrm{I}}{c_{1}} \mathrm{si} \mathrm{C}_{1}<0$, supérieure à $\mathrm{C}_{2}$ et $\frac{\mathrm{I}}{c_{2}} \mathrm{si} c_{2}<0$, ou comprise dans les intervalles correspondants si, respectivement, $\mathrm{C}_{1}$ ou $c_{2}$ est positif.

- Dans tous les cas, il existe donc au moins une fréquence d'équilibre. Il n'y en a qu'une, car on vérifie que $\alpha$ et $\beta$ sont tous deux inférieurs à $r$, ce qui empêche la condition (2) d'être remplie.

On peut montrer, dans des cas particuliers, que cet équilibre unique est stable et cela est de toute façon suggéré par le fait que les équilibres correspondant à la fixation de l'un ou l'autre homozygote sont instables.

e) Overdominance positive dans un sexe, négative dans l'autre.

$\begin{array}{lllll}\text { Prenons } & \mathrm{S}_{1}>0 & s_{1}>0 & \mathrm{~S}_{2}<0 & s_{2}<0 . \\ \text { D'où } & \mathrm{C}_{1}<\mathrm{I} & c_{1}<\mathrm{I} & \mathrm{C}_{2}>\mathrm{I} & c_{2}>\mathrm{I} .\end{array}$

Comme $c_{2}>0$, une racine de $f(t)=0$, pour correspondre à un équilibre réel, doit être comprise dans l'intervalle $\left(\mathrm{C}_{2}, \frac{\mathrm{I}}{c_{2}}\right)$.

Sans perte de généralité, nous pourrons supposer $C_{2}>c_{2}$.

Le tableau 6 considère les dispositions relatives possibles de $c_{1}, \frac{I}{C_{1}}, C_{2}, \frac{I}{c_{2}}$, le signe de $f(t)$ pour chacune de ces valeurs, d'où les possibilités d'équilibres, en distinguant le cas $C_{1}>0$ (une racine "valable " devant être comprise dans l'intervalle $c_{1}-\frac{\mathrm{I}}{\mathrm{C}_{1}}$ ) et $\mathrm{C}_{1}<\mathrm{o}$ (racine devant être supérieure au plus grand des deux nombres $c_{1}, \frac{\mathrm{I}}{\mathrm{C}_{1}}$ ).

Nous avons vérifié numériquement la possibilité de trois équilibres, en particulier, dans le cas $2 a$ : les deux exemples donnés par OwEN (r953) y correspondent (du moins à la limite), le premier avec les valeurs $\mathrm{C}_{1}=c_{1}=0, \mathrm{C}_{2}=c_{2}=3,3$, le deuxième avec les valeurs $\mathrm{C}_{1}=c_{1}=0, \mathrm{C}_{2}=7,2, c_{2}=6,0$.

L'exemple C du tableau 3 de BoDmer (I965, p. 4I8) est analogue. Notre cas I $a$ est illustré par l'exemple de $\mathrm{L}_{1} \mathrm{I}\left(\mathrm{r} 967, \mathrm{p}\right.$. 47 I) avec $c_{1}=\mathrm{C}_{1}=3,67, c_{2}=$ $\mathrm{C}_{2}=-0,067$. Par contre, l'exemple $\mathrm{D}$ du tableau 3 de BoDMER correspond à un seul équilibre (stable) pour notre cas $2 a$.

Un exemple numérique à deux équilibres peut être donné pour le cas $\mathrm{I} b$, avec $\mathrm{C}_{1}=-0,5 ; c_{2}=+\mathrm{I}, 5 ; \quad \mathrm{C}_{2}=+4,0 ; c_{1}=+0,75$. La zone acceptable pour une racine correspondant à un équilibre est comprise entre $+0,75$ et +4 , et il y a trois racines positives, l'une inférieure à $\frac{2}{3}$, la seconde égale à $I$, la troisième comprise entre 2 et 3 . Les deux dernières répondent donc à la question. 
TABLEAU 6

Possibilités d'équilibre: cas oì il y a overdominance positive dans un sexe, négative dans l'autre

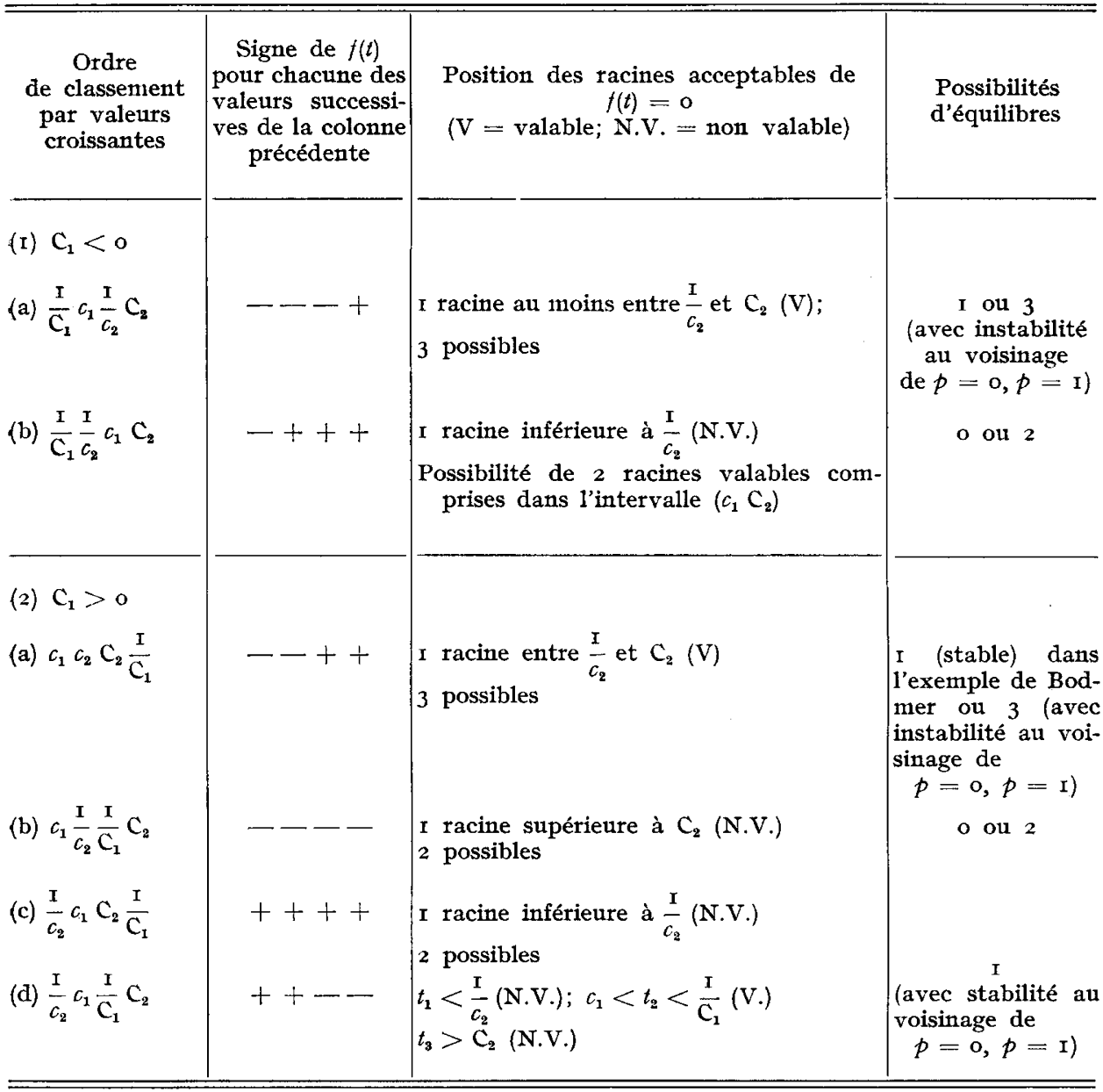

Avec $\mathrm{C}_{1}=\frac{\mathrm{I}}{2,9}, c_{1}=0, \mathrm{C}_{2}=3, c_{2}=5$, on réalise deux équilibres dans notre cas $2 b$; 1'exemple $\mathrm{B}$ du tableau 3 de BODMER conduit à la même conclusion et rentre dans le cas $2 \mathrm{C}$.

Enfin, l'exemple A du tableau 3 de BoDMER illustre le cas $2 d$ avec un seul équilibre instable.

En fin de compte, s'il y a overdominance positive dans un sexe et négative dans l'autre, on peut avoir zéro, un, deux ou trois équilibres.

f) Pas d'overdominance dans un sexe, overdominance négative dans l'autre.

On a alors, par exemple : $\mathrm{C}_{1}>\mathrm{I} \quad c_{1}>\mathrm{I} \quad \mathrm{C}_{2}<\mathrm{I} \quad c_{2}>\mathrm{I}$.

Une racine de $f(t)=0$ correspondant à un équilibre doit être comprise dans 
l'intervalle commun à $\left(c_{1}-\frac{I}{C_{1}}\right)$ et à $\left(\frac{I}{c_{2}}-C_{2}\right), C_{1}$ et $c_{2}$ étant positifs. Pour que cet intervalle existe, il faut que $C_{2}, \frac{I}{c_{2}}$ ou les deux, soient supérieurs à $\frac{I}{C_{1}}$.

Dans tous les cas, une seule racine est possible, ou deux, mais non trois, dans l'intervalle requis, tout entier inférieur à $I$, puisque le produit des racines est $I$.

Le tableau 7 indique, comme précédemment, les divers cas possibles (on peut noter, en plus, que $f(+\mathrm{r})$ est toujours négatif).

\section{TABLEAU 7}

Possibilités d'équilibre: cas où il n'y a pas overdominance dans un sexe, overdominance négative dans l'autre

\begin{tabular}{|c|c|c|c|}
\hline $\begin{array}{c}\text { Ordre } \\
\text { de classement } \\
\text { par valeurs } \\
\text { croissantes }\end{array}$ & $\begin{array}{c}\text { Signe de } f(t) \\
\text { pour chacune des } \\
\text { valeurs successi- } \\
\text { ves de la colonne } \\
\text { précédente }\end{array}$ & $\begin{array}{c}\text { Position des racines de } f(t)=o \\
(\mathrm{~V}=\text { valable; } \mathrm{N} . \mathrm{V} .=\text { non valable })\end{array}$ & $\begin{array}{l}\text { Possibilités } \\
\text { d'équilibre }\end{array}$ \\
\hline (a) $\mathrm{C}_{2} \frac{\mathrm{I}}{\mathrm{C}_{1}} \frac{\mathrm{I}}{c_{2}} c_{1}$ & $--\cdots$ & $\begin{array}{l}\text { I racine }>c_{1} \text { (N.V.); } 2 \text { racines valables } \\
\text { possibles }\end{array}$ & o ou 2 \\
\hline (b) $\frac{\mathrm{I}}{c_{2}} \frac{\mathrm{I}}{\mathrm{C}_{1}} \mathrm{C}_{2} c_{1}$ & $++\cdots$ & $\begin{array}{l}t_{1}<\frac{\mathrm{I}}{c_{2}}(\mathrm{~N} . \mathrm{V} .) ; \frac{\mathrm{I}}{\mathrm{C}_{1}}<t_{2}<\mathrm{C}_{2}(\mathrm{~V}) \\
t_{3}>c_{1} \text { (N.V.) }\end{array}$ & I \\
\hline (c) $\frac{\mathrm{I}}{\mathrm{C}_{1}} \frac{\mathrm{I}}{c_{2}} \mathrm{C}_{2} c_{1}$ & ++-- & $\begin{array}{l}t_{1}<\frac{\mathrm{I}}{\mathrm{C}_{1}}(\mathrm{~N} . \mathrm{V} .) ; \frac{\mathrm{I}}{c_{2}}<t_{2}<\mathrm{C}_{2}(\mathrm{~V}) \\
t_{3}>c_{1} \text { (N.V.) }\end{array}$ & $\mathbf{I}$ \\
\hline (d) $\frac{I}{C_{1}} C_{2} \frac{I}{c_{2}} c_{1}$ & $++\cdots$ & $\begin{array}{l}t_{1}<\frac{\mathrm{I}}{\mathrm{C}_{1}}(\mathrm{~N} . \mathrm{V} .) ; \mathrm{C}_{2}<t_{2}<\frac{\mathrm{I}}{c_{2}}(\mathrm{~V}) \\
t_{3}>c_{1} \text { (N.V.) }\end{array}$ & I \\
\hline
\end{tabular}

On a donc, dans cette situation, 0 , I ou 2 équilibres possibles. L'existence de deux équilibres se vérifie numériquement, par exemple, avec $\mathrm{C}_{1}=5 ; c_{1}=\mathrm{x}, 2$; $\mathrm{C}_{2}=0 ; c_{2}=\mathrm{I}, 25$. Il y a alors deux racines acceptables, l'une supérieure à 0,2 , l'autre inférieure à 0,8 .

g) Overdominance négative dans les deux sexes.

Tous les paramètres $\mathrm{C}$ sont alors supérieurs à $+\mathrm{r}$. Les deux intervalles $\left(c_{1}, \frac{I}{C_{1}}\right)$ et $\left(C_{2}, \frac{I}{c_{2}}\right)$ ont nécessairement une partie commune englobant $\mathrm{I}$.

De la même manière que précédemment, on montre que $f(t) \geqslant 0$ pour $t=\frac{\mathrm{I}}{\mathrm{C}_{\mathbf{1}}}$ ou $t=\frac{\mathrm{I}}{c_{2}}$ et $f(t)<\mathrm{o}$ pour $t=c_{1}$ ou $t=\mathrm{C}_{2}$. Il y a donc nécessairement une racine comprise dans l'intervalle cherché. 
Comme $f(0)=-$ I et $f(+\infty)=+\infty$, on voit qu'il y a toujours, dans ce cas, trois racines positives, l'une comprise entre o et la plus petite des valeurs $\frac{\mathrm{I}}{\mathrm{C}_{1}}$ et $\frac{\mathrm{I}}{c_{2}}$, la seconde entre la plus grande de ces deux valeurs et la plus petite de $c_{1}$ ou de $\mathrm{C}_{2}$, la troisième supérieure à la plus grande des deux valeurs $c_{1}$ ou $\mathrm{C}_{2}$. Seule, la deuxième racine correspond à un équilibre réel. Il y a donc, dans ce cas, toujours un équilibre et un seul. Il est facile de montrer, sur des exemples numériques, que cet équilibre est instable : cela est aussi suggéré par le fait que les équilibres correspondant à la fixation des deux homozygotes sont stables.

\section{CONCLUSION}

Les possibilités d'équilibres (stables ou instables) des fréquences géniques dans les diverses situations passées en revue pour un locus autosomal avec sélection différente dans les deux sexes sont donc les suivantes :

- Absence d'overdominance : aucun équilibre si le classement des génotypes est identique dans les deux sexes; 0 , I ou parfois 2 équilibres (avec possibilité d'un stable et d'un instable) si le classement des homozygotes s'inverse d'un sexe à l'autre. Nous suggérons dans le tableau 4 que l'équilibre, quand il est unique, peut être stable ou instable, suivant le cas, à partir de la prise en considération de la stabilité des équilibres correspondant à la fixation de $\mathrm{A}$ ou de $a$.

- Overdominance positive dans un sexe, absente dans l'autre : o ou I équilibre.

- Overdominance positive dans les deux sexes : I équilibre (dont la stabilité est suggérée par l'instabilité au voisinage de $p=0$ ou $p=\mathrm{I}$ ).

- Overdominance positive dans un sexe, négative dans 1'autre : $0, I, 2$ ou 3 équilibres. L'équilibre unique doit pouvoir être stable ou instable à en juger par une discussion de la stabilité des équilibres correspondant à la fixation de A ou de $a$. Dans le cas de trois équilibres, deux peuvent être stables.

- Overdominance absente dans un sexe, négative dans l'autre, 0 , I ou 2 équilibres.

- Overdominance négative dans les deux sexes : I équilibre (dont l'instabilité est suggérée par la stabilité au voisinage de $p=0$ ou $p=\mathrm{I}$ ).

Ce tableau d'ensemble vérifie, dans le cas général. les possibilités trouvées par BODMER (I965) dans une situation particulière.

Il montre que l'existence de trois équilibres n'est possible que lorsque l'hétérozygote est favorisé dans un sexe et défavorisé dans l'autre par rapport aux deux homozygotes.

Il confirme la possibilité de deux équilibres montrée par BoDmer. Ainsi, un équilibre stable peut exister, qui ne serait pas atteint à partir d'une population fixée homozygote $a a$ par mutation vers $\mathrm{A}, a a$ étant aussi un équilibre stable.

Ces équilibres, obtenus lorsque la sélection diffère entre sexes, peuvent posséder des propriétés assez particulières : ainsi, dans la situation d'équilibre, il y a persistance possible d'une variabilité "additive "importante liée au locus en cause. 
D'autre part, dans le cas de populations d'effectif limité, il serait intéressant de rechercher l'éventualité de variations ou oscillations d'apparence brusque, associées à la présence d'un équilibre instable, ou de deux ou trois équilibres.

De tels phénomènes seraient susceptibles d'apporter, à l'occasion, des perturbations imprévues dans le résultat de la sélection artificielle pour un caractère métrique dépendant de gènes à effet influencé par le sexe.

Reçu pour publication en janvier 1968.

\section{REMERCIEMENTS}

Nous tenons à remercier vivement le Pr LETORT, de l'Institut national agronomique, Paris, des critiques et suggestions très utiles qu'il nous a apportées en lisant ce manuscrit

\section{SUMMARY}

SELECTION DIFFERING IN MALES AND FEMALES.

GENERAL, DISCUSSION OF THE POSSIBILITIES OF EQUILIBRIUM FOR AN AUTOSOMAL, LOCUS

WITH TWO ALLELES

A survey is presented, for the general case, of the possible equilibria of gene frequencies, for an autosomal locus with two alleles in a panmictic population, when selective values differ in the two sexes. The possibilities are the following :

- No overdominance : no non-trivial equilibrium if the ranking of the genotypes is identical in both sexes; 0,1 or 2 equilibria if the ranking of the homozygotes is reversed from one sex to the other. When it is unique, the equilibrium may be stable or unstable as suggested by a discussion of the stability near the fixation of $\mathrm{A}$ or $\boldsymbol{a}$.

- Positive overdominance in one sex, no overdominance in the other : 0 or 1 equilibrium.

- Positive overdominance in both sexes : 1 equilibrium (stable as suggested by the instability near the fixation of $A$ or $a$ ).

- Positive overdominance in one sex, negative overdominance in the other; $0,1,2$, or 3 equilibria.

- No overdominance in one sex, negative overdominance in the other : 0,1 or 2 equilibria.

- Negative overdominance in both sexes : 1 equilibrium (unstable as suggested by the stability near the fixation of $\mathrm{A}$ or $a$ ).

The results confirm, in particular, the possibility of two equilibria of which one may be stable. They show that the realization of three equilibria, of which two may be stable, is possible only when the heterozygote is favoured in one sex and at a disadvantage in the other with respect to the two homozygotes.

\section{RÉFÉRENCES BIBLIOGRAPHIQUES}

BenNetr H. H., r958. The existence and stability of selectively balanced polymorphisms at a sex linked locus. A ust. J. biol. Sci., 11, 598-602.

BODMER W. F., 1965. Differential fertility in population genetics models. Genetics, 51, 4 11-424.

HALdANE J. B. S., I 924 A mathematical theory of natural and artificial selection. I. Trans. Cambr. Philos. Soc., 23, I9-4r.

Haldane J. B. S., I926. A mathematical theory of natural and artificial selection III. Proc. Cambr. Philos. Soc., 23, 363-372.

Haldane J. B. S., 1962. Conditions for stable polymorphism at an autosomal locus. Nature, 193, I 108. HALDANE J.B.S., JAYAKaR S. D., I964. Equilibria under natural selection at a sexlinked locus. J. Genet., $59,29-36$. 
I, C. C., 1963. Equilibrium under differential selection in the sexes. Evolution, 17, 493-496.

I, C.C., r967. Genetic equilibrium under selection. Biometrics, 28, 397-484.

MANDEL S. P. H., r959. Stable equilibrium at a sex linked locus. Nature, 188, r347-1348.

Mrrat P., 1966. Effets maternels et évolution de la fréquence d'un gène. Ann. Biol. anim. Bioch. Biophys., 6, $521-528$.

MÉrat P., 1967. Les gènes influant sur la variance d'un caractère quantitatif et leurs répercussions possibles sur la sélection. Ann. Génét., 10, 21 2-220.

OWEN A. R. G., 1953. A genetical system admitting of two distinct stable equilibria under natural selection. Heredity, 7, 97-102.

PARsons P. A., r96r. The initial progress of new genes with viability differences between sexes and with sex linkage. Heredity, 16, I03-xo7.

Sprorr D. A., r957. The stability of a sex-linked allelic system. Ann. hum. Genet., 22, 1-6. 